\title{
ARCHITECTURE OF PROVIDING SERVICES IN INTELLIGENT TRANSPORTATION SYSTEMS USING SEMANTICALLY DEFINED INFORMATION
}

Intelligent transportation systems (ITS) is an area currently developing very fast. The key to the ITS success will definitely be the implementation of services which would be solving all of the requirements of the road transportation participants. The article proposes the architecture to be used to control the services provided within the intelligent transportation systems. The architecture presupposes using semantically modelled information to be able to find new services in more intelligent way, to preliminarily distinguish unknown services and use them anyway.

\section{Introduction}

Transportation in the European Union is becoming more and more important component of the economy. Every year more and more passengers and cargo are to be transported. As the Document by the European Committee 'Keep Europe moving - Sustainable mobility for our continent' [1] reads, the volume of cargo transportation grew by $2.8 \%$ every year from 1995 to 2004 . The passenger transportation growth rate was $1.9 \%$. Between 1995 and 2004 . the cargo transportation grew by $28 \%$, and the passenger transportation grew by $18 \%$ in total.

These facts say that the growth is regular, and the tendency does not seem to be changing. Unfortunately, the transportation infrastructure is not developing equally quickly. Apart from other consequences, this has also been resulting into a number of car accidents on overly busy roads, with lots of lost human lives and much time wasted in traffic jams. All these facts sum up into huge economic loss and, last but not least, they represent damages to the environment.

It is not easy at all to keep adding up new and new infrastructure, or, eventually, extending the existing infrastructure, and thus hightening its capacity. Solutions like these are economically very demanding, as well as sources are limited (both space and time-wise). One of the possibilities is to involve informatics into the process of solving these problems, and help achieve efficient and intelligent control of the existing transportation and its systems. The intelligent transportation systems (ITS) can organize and manage transportation systems in such way that they can be used as efficiently and economically as possible.

\section{Communication in the intelligent transportation systems}

Transportation systems are a specific type of systems, namely from the viewpoint of communication networks. It is not possible to fully apply the knowledge and principles used in common computer networks. With computer networks the infrastructure is usually fixed or localized in relatively small geographical locations, whereas in the ITS the networks are geographically vast, and usually they are not even interconnected completely. Moreover, contrary to common networks, where the equipment is usually known in advance (computers, routers, portals etc.) and also the services and their communication protocols are known (WWW - HTTP, VOIP - SIP, E-mail - SMTP, ...), in the transportation systems networks the equipment is considerably different - transportation infrastructure, intelligent signs, parking systems, toll systems, garages, motor-cars etc.

The services the above agents involved are able to provide are diverse (information on navigation, fees, traffic jams on roads, parking vacancies, defined traffic diversions ...). In opposition to traditional networks, the services need not be known in advance. The standards given for this area are only being created, and the question remains to what extent the producers will be willing to implement them, or whether they will rather use their own proprietary systems, which can give them an advantage on the market.

In Europe it is the European Telecommunications Standards Institute (ETSI) that creates standards for the area. Since it deals mainly with the standards for the area of information and communication technologies, most of the standards are oriented to this area also in the ITS. Among the standards (most of which are just being designed) there are standards concerning the commu-

\footnotetext{
* Patrik Hrkut

Department of Software Technology, Faculty of Management Science and Informatics, University of Zilina, Slovakia,

E-mail: patrik.hrkut@fri.uniza.sk
} 
nication between a vehicle and the infrastructure, such as Dedicated Short-Range Communications (DSRC), Continuous Air Interface Long and Medium Range (CALM), as well as the mutual communication between vehicles themselves [2]. They are standards that prevailingly resolve especially lower levels of communication, but it is also possible to find some standards describing potential applications in the ITS area [3]. What is also promising is the initiative of the motor-car (Audi, Fiat, Opel, Volvo, Volkswagen a others) producers and other companies that have established CAR 2 CAR Communication Consortium [4]. The main aims of the Consortium are as follow:

- developing and pursuing the standards in the ITS area

- creating foundations and specifications for the standards, especially in cooperating with the ETSI,

- contributing to the worldwide harmonisation of the $\mathrm{C} 2 \mathrm{C}$ standards,

- supporting the unified implementation of an exclusive communication frequency ,

- creating realistic strategies and business models of the $\mathrm{C} 2 \mathrm{C}$ applications implementation,

- demonstrating possibilities and feasibility of the $\mathrm{C} 2 \mathrm{C}$ applications.

The architecture this article suggests to use is not proposed to be an alternative to the above standards, it is rather an idea of generalising some of the application protocols so that the interoperability between various participants of communication can become easier, and new services on the same protocol platform of lower levels can be developed and distinguished in more simple way.

\section{Services in intelligent transportation systems}

The basic categorisation of the ITS services is concentrating on the participants of communication. According to which parties participate in the communication, the services have been divided into two essential categories as follow:

- communication between a vehicle and the infrastructure (Vehicle 2 Infrastructure, V2I),

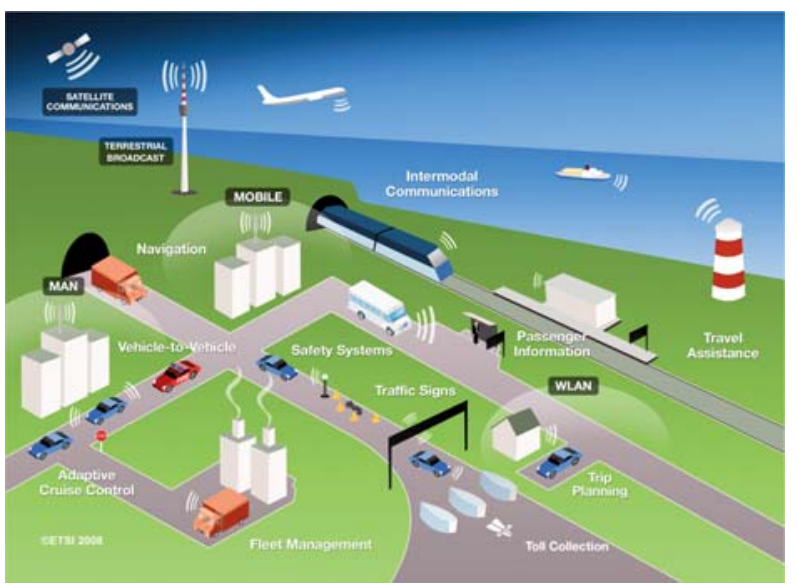

Fig. 1 Types of communication in the ITS
- communication between vehicles (Vehicle 2 Vehicle, V2V, sometimes also Car 2 Car, $\mathrm{C} 2 \mathrm{C}$ ).

The communication does not necessarily have to be focussed only on the road transportation participants, but in general all kinds of transportation can communicate with each other. Figure 1 from ETSI website also shows that not just the road infrastructure can serve as the source of information, but it can also be any other device able to provide information in one or both directions (satellite, mobile networks).

The architecture the article presents has been designed in a general way, with no ties to any particular communication type. Its main use can be seen in the mutual communication among vehicles (e.g. the exchange of multi-media files among vehicles), but also in the communication between a vehicle and the infrastructure (e.g. reporting dangerous situations on the road).

As it has been specified in the above part, the standardising process in progress is concentrating mainly on lower communication levels. This is understandable because without the lower level standards, the communication is not possible at all. However, it is equally important to agree on the way the services to be provided in the ITS.. The services are the key to success of the intelligent transportation services. Therefore it is very important that motorcar producers agree on a group of services the devices will support. The unified transmission zone will not guarantee that a vehicle understands every information that gets to it. The architecture proposed hereby tries to solve the following questions:

- how to sort out the communication between the devices, when one of them is offering an unknown service, or a service which the other device does not recognize as it has not been defined precisely,

- how to complement an existing service with a new dimension or property which are not directly supported by the other device,

- how to update the services without having to change the software of all of the devices.

\section{Semantic modelling of information}

In 2001 the web founder Timothy Berners-Lee expressed his idea of further routing the WWW service on the internet. The web contains a great lot of text and other information that are easy to access for people, but machines (other computers) are not able to categorise or search for them. As the information does not contain any other complementary information or its ties to other information, machines cannot understand and connect mutually related information. Searching is therefore limited just to key words, and other information having semantic ties to the information are not found.

Metainformation supplying to the existing information, or, in other words, to annotating the existing text could be the solution. These processes can be automated to a certain extent, but only the person who arranges the pre-processed text apropriately can supply the information with real meaning. 
Several languages have been used to make formal records of the relations among information. The most frequently used ones are RDF, RDF Scheme and OWL. The following chapters describe the languages briefly, in order to introduce the corner-stones of the proposed architecture.

\section{RDF language}

The RDF language [5] is intended to represent the information on the sources in the WWW environment. It was originally used to describe metadata, such as author, title, time, licence information etc. The language is also possible to use in applications other than it was originally meant for. The RDF data model is based on triples of elements: subject, predicate and object, which all together create a statement (declaration). The source can serve as the subject or object, and the predicate corresponds to the object attributes that have been observed. The model represents the triple: the subject has an attribute (predicate) of a certain value (object)

There is no defined representation for the RDF. You can use a graph representation, as well as an ordinary text description. The $\mathrm{W} 3 \mathrm{C}$ has issued a recommendation for the serialisation based on the XML language, i. e. sequencing the individual statements into elements and attributes of the XML language.

Here is an example of a record in the XML language:

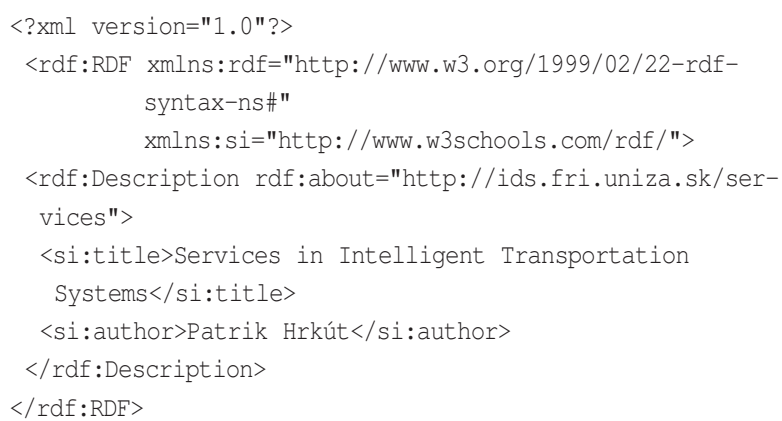

The same example written down in the form of a graph:

\section{RDF Scheme}

The RDF language describes sources by means of classes, attributes and values; it needs a tool to describe application-specific classes and attributes. The RDF Scheme is a semantic extention of the RDF, and it provides mechanisms to describe the related sources groups and relations among them. The RDF Scheme data model enables to create data classes. The system of classes and attributes is very similar to the object-oriented languages. A class is defined as a sources group with identical characteristics. The classes can create hierarchies on the basis of the relations. The RDF Scheme does not provide dictionaries for applications, but it can describe relevant classes and attributes and their mutual relations.

\section{Ontologies}

Ontologies represent the core of semantic information modelling. Before the applications are able to understand each other and to recognize information in the data, it is necessary for them to use mutually compatible data descriptions, i. e. to abide with syntactical inter-operability. This way the systems will understand the contents of documents correctly.

The term 'ontology' originates in philosophy, and it denotes a theory of being, of what exists, and what are the relations between the particular elements. Ontologies usually include definitions of classes, relations among them, functions and restrictions. A typical ontology contains a hierarchical description of important notions of a given domain.

In the area of the intelligent transportation services it is possible to create an ontology, and to use it to define a common dictionary to be used by all the participants of the communication in the ITS. It will be necessary to collect all notions and define their mutual relations. Then, on the basis of the collected information, conveniently inter-related on the grounds of their semantic reciprocality, it will be possible to create a source of information on not just the services provided in the ITS but also generally on all terms from this specific area. At the end, it will be necessary to write the information down by means of a formal language. A suitable candidate is the OWL language.

\section{OWL language}

The OWL contains more options of describing attributes and classes than the RDF and RDF Scheme do; apart from other things it can describe relations among classes, such as disjunction, equivalence or cardinality. At the same time it contains wider possibilities for the description and characterisation of attributes. The language was designed to be used by the applications which need to process the contents of documents. The OWL guarantees mutual interconnectivity of the contents, and provides good possibilities for describing the relations among information. The OWL has three variants differing from each other by their expressing abilities (in downgoing sequence) - OWL Full, OWL DL and OWL Lite. The OWL DL is the most frequently used one.

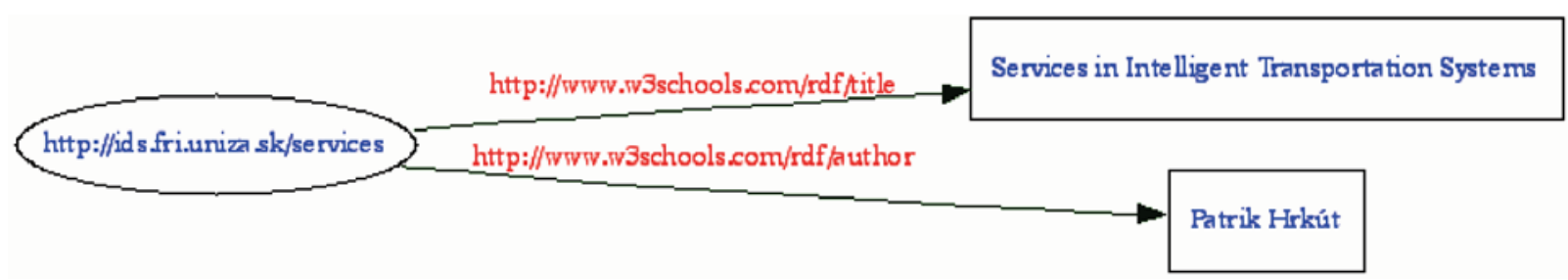

Fig. 2 The RDF statement written down in the form of a graph 
With its expression rate, the OWL DL draw near the OWL Full, with one difference - a statement evaluation is guaranteed within definite time. The OWL DL supports a set of constructions identical with the one the OWL Full does, with one difference: it does not support free mixing of the RDF and RDF Scheme, and it requires a strict separation of classes, individualities (in the notions of the object-oriented programming, an individuality is understood as a class instance), attributes and values (a class can be a sub-class to other classes, but it cannot be an instance to another class).

\section{Design of the architecture to provide the ITS services}

As it has been mentioned already, the suggested architecture is aiming at covering various types of services in the ITS. In the area developing as quickly as the ITS is, there will always be new services appearing, or, the existing services will be enriched with new attributes. One of the questions arising here is how to incorporate the new services and attributes into the existing systems without having to upgrade the existing devices in any remarkable way, whether they are devices in vehicles or in road infrastructure. One of the things the suggested architecture is supposed to do is to simplify the creation of new services as much as possible, as well as to simplify their registration and distribution to the devices.

The essence of the architecture is supposed to consist of the ITS area ontology, which should combine all of the substantial notions of the domain. The primary contents of the ontology would consist of:

- vehicles, categorisation and attributes,

- road infrastructure elements,

- services and their characterisations,

- events, car accidents, dangerous situations.

Each newly originated service will be described in detail, categorised and suitably incorporated into the existing ontology. The ontology like this could be distributed (e.g. using distributed databases [6]) in the environment of road infrastructure or over the internet. In case a vehicle gets within the reach of a device providing newer definitions of services, it would automatically update its definitions of services. Of course, other vehicles disposing of the newer ontology version are also able to convey the information, and vice versa, vehicles with the latest ontology version would be spreading it.

It is important to make sure the ontology is possible to be used on the devices in the ITS. It is not possible to expect that the devices would dispose of a remarkable calculation performance, or of an extensive memory for complicated questioning which has been done over ontologies. To solve this problem, we could use a transformation, e. g. the XSLT, so that we can simplify the information model in such a way that the information can be used for simpler devices. Another potential solution would be implementing a transformation or a simplified query language which would be optimised for the devices with a weaker calculation performance.

The following example will show us what the process might look like in practice: a vehicle is signalling to another vehicles coming from the opposite direction that they are heading towards a road section where there is a dangerous situation. Not all vehicles are able to understand that information exactly, but it is quite enough if they are able to file it into a correct category, and, on the basis of detailed information they have in their ontology, they are able to estimate the type of the received information and its importance, in spite of the fact that they do not recognize it.

Last but not least, it is important to ensure authenticity and unchangeability of the provided information, whether the information comes from a source of the infrastructure or from another vehicle. To solve such problems in practice, we often use the Public Key Infrastructure (PKI), where the information is signed with a private key of the issuer owning a credible certificate of a certification authority. The information receiver may verify the information by the information sender's public key. More information on the PKI implementation can be found in [7].

\section{Conclusion}

The advantages of the proposed architecture will make it easier to control the existing services, faster to implement new services, more comfortable to incorporate services into the existing systems. The architecture should bring more intelligence into managing the services in the ITS. At the moment, the submitted architecture is being designed, and the selection of the IT area technologies to be used is being finalised. The pilot verification of the proposed architecture is going to take place within the Easyway Project which Slovakia has been participating in.

\section{Acknowledgement:}

This contribution is the result of the project implementation: Centre of excellence for systems and services of intelligent transport, ITMS 26220120028 supported by the Research \& Development Operational Programme funded by the ERDF.

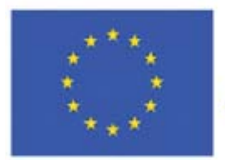

Európska únia
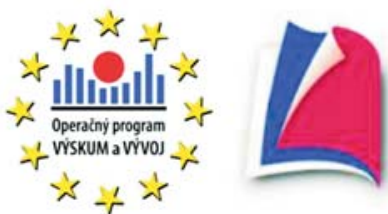

Agentúra

Ministerstva školstva, vedy, výskumu a športu SR pre štrukturálne fondy EÚ

"Podporujeme vyskumne aktivity na Slovensku/Projekt je spolufinancovany zo zdrojov EU." 


\section{References}

[1] Communication from the commission to the council and the european parliament Keep Europe moving - Sustainable mobility for our continent, Mid-term review of the European Commission's 2001 Transport White Paper. 2006. Brussels. Available from: $\mathrm{http}$ ://ec.europa.eu/transport/transport_policy_review/doc/2006_transport_policy_review_en.pdf

[2] EN 300674 Road Transport and Traffic Telematics (RTTT); Dedicated Short Range Communication (DSRC) transmission equipment (500 kbit/s / $250 \mathrm{kbit} / \mathrm{s})$ operating in the 5,8 GHz Industrial, Scientific and Medical (ISM) band. Available from: $\mathrm{http}$ ///webapp.etsi.org/workprogram/Frame_WorkItemList.asp?SearchPage=TRUE\&qETSI_STANDARD_TYPE=\&qETSI_NU MBER $=300+674$

[3] ETSI TR 102638 Intelligent Transport Systems (ITS); Vehicular Communications; Basic Set of Applications; Definitions. Available from: http://pda.etsi.org/pda/home.asp?wki_id=E-GA9-A_UJ46C974vO.qm

[4] Car 2. Car Consortium, Main page, http://www.car-to-car.org/

[5] RDF Primer, W3C Recommendation. Available from: http://www.w3.org/TR/2004/REC-rdf-primer-20040210/

[6] JANECH, J., BACA, T.: Distributed Database Systems in Vehicular Ad-hoc Network, In: Communications - Scientific Letters of the University of Zilina, No. 1, 2010, ISSN 1335-4205.

[7] KRSAK, E., MARTINCOVA, P.: Digital Signature Implementation in Educational Institutions, Intern. conference on Information Technologies (InfoTech-20009), 2009, Varna, Bulgaria. In: Proc. of the 23rd International Conference on Systems for Automation of Engineering and Research (SAER-2009 ), Sofia : State Agency for Information Technology and Communication : Technical University of Sofia, 2009. - ISBN 978-954-439-771-6, pp. 60-67. 